\title{
Relações entre suporte social, autorregulação e consumo de outras substâncias em adultos portugueses
}

\author{
Relationships between Social Support, Self-Regulation \\ and Drug Consumptions among Portuguese Adults
}

\author{
José Manuel Borges ${ }^{1}$ \\ Jose A. García del Castillo \\ Juan Carlos $\mathrm{Marzo}^{3}$ \\ Álvaro García del Castillo-López ${ }^{4}$ \\ Universidad Miguel Hernández, España
}

Resumo. Neste estudo, investigaram-se as relações entre suporte social percebido, auto-regulação e comportamentos de consumo de álcool, tabaco e outras substâncias psicoactivas. A amostra foi constituída por 340 participantes. O suporte social correlacionou-se favoravelmente com a auto-regulação, mas somente na vertente de satisfação com o apoio social é que se observou esta tendência de forma evidente. $\mathrm{O}$ número de figuras de apoio diferenciou-se significativamente com o uso de tabaco, o que sugere o âmbito afiliativo de algumas substâncias psicoactivas. Nesse sentido, o uso de tabaco pode estar associado a uma rede social mais ampla, mas não com a qualidade do suporte social.

Palavras chave. Suporte social, autorregulação, comportamentos de uso, tabaco, outras substâncias.

Abstract. In this study the relationships between perceived social support, self-regulation and consuming behaviors of tobacco and other psychoactive substances we investigated. The sample consisted of 340 adult participants. Social support correlated positively with self-regulation, but was solely in the aspect of satisfaction with social support that this trend was noted clearly. The number of support providers differed considerably with the use of tobacco, which suggests the affiliative scope of some psychoactive substances. Accordingly, the use of tobacco may be associated with a larger social network, but not with the quality of social support.

Keywords. Social support, Self-regulation, Use behaviors, Tobacco, Other substances.

\footnotetext{
${ }^{1}$ José Manuel Borges. Universidad Miguel Hernández, España. Dirección postal: Carretera de Alicante-Valencia s/n. 03550-Sant Joan d'Alacant (Alicante) España.. E-mail: josesardinhaborges@hotmail.com

${ }^{2}$ Jose A. García del Castillo. Universidad Miguel Hernández, España. E-mail: jagr@umh.es

${ }_{3}^{3}$ Juan Carlos Marzo. Universidad Miguel Hernández, España. E-mail: jc.marzo@umh.es

${ }_{4}^{4}$ Álvaro García del Castillo-López. Universidad Miguel Hernández, España. E-mail: alvaro.garciac@umh.es
}

\section{@ $\odot \Theta \Theta$}

Esta obra está bajo una licencia de Creative Commons Reconocimiento-NoComercial-SinObraDerivada 4.0 Internacional. 


\section{Introdução}

A autorregulação apresenta-se, presentemente, como uma competência humana tão intensamente investigada quanto especulada, possivelmente a mais fundamental (Zimmerman, 2000). Trata-se também de um termo generalista usado comumente para descrever os diversos processos psicológicos que possibilitam o estabelecimento de objetivos (Mischel, Cantor \& Feldman, 1996). Os objetivos são representações mentais de desenlaces desejados pelas pessoas que se comprometem com os mesmos (Fujita \& MacGregor, 2012). Estabelecer um objetivo não se cinge apenas à intenção de alcançar um valor. Muitos obstáculos podem ser encontrados na transformação de uma vontade vaga em uma meta bem definida (Norcross, Ratzin \& Payne, 1989). Por exemplo, muitas pessoas gostariam de ser muito saudáveis, mas menos estão dispostas a comer fruta e vegetais com a frequência necessária para atingir esse resultado. Logo, o estabelecimento de objetivos implica um sentimento de comprometimento ou uma sensação de urgência que motiva os sujeitos a operacionalizarem esforços concretos no sentido de reduzir a discrepância entre o seu estado actual e o estado desejado (Carver \& Scheier, 1982).

Complementarmente, o autocontrolo é a aptidão para resistir aos impulsos, sendo muito importante no auxílio à orientação voluntária do comportamento (Baumeister, Vohs \& Tice, 2007; Carver \& Scheier,1998) e parece constituir um componente de autorregulação importante no estudo do uso de substâncias (Neal \& Carey, 2005).

A influência das competências de autorregulação sobre os comportamentos de uso de substâncias tem sido constatada em variados grupos populacionais e culturas (Neal \& Carey, 2005). Nesse sentido, as investigações mais recentes parecem indicar a dificuldade no auto-controle enquanto um mecanismo intermédio entre a vulnerabilidade biológica e a escalada no abuso de substâncias psicoactivas (Glantz \& Leshner, 2000).

$\mathrm{Na}$ adultícia, é esperada autonomia regulatória, mas não totalmente, porque o Ser Humano maduro solicita sempre ajuda, partilha, motivação e apoio emocional das relações interpessoais chegadas que tem ao seu dispor (Goetz, Keltner \& Simon-Thomas, 2010). Por outro lado, uma competência autorregulatória de qualidade pode ser conotada com relações interpessoais mais significativas (Busch \& Hofer, 2012).

O apoio social percebido é vulgarmente concebido como o nível em que um indivíduo aprecia as suas relações sociais enquanto disponíveis para lhe prover ajuda em caso de necessidade (Sarason, Sarason \& Pierce, 1990). Uma característica fundamental da perceção do suporte social é que esta se concentra nas necessidades sociais básicas do indivíduo (de afeto, de identificação, de pertença, de segurança ou de aprovação) e até que ponto estas são satisfeitas através da interação com os outros (Thoits, 1986).

O papel do apoio social nos comportamentos de consumo figura-se ambíguo (Cullum, O'Grady, Sandoval, Armeli \& Tennen, 2013): Por um lado, a título de exemplo, estudantes universitários, com grande percentagem de familiares integrados nas suas redes sociais, assinalaram níveis mais baixos de ingestão de bebidas alcoólicas (Reifman, Watson \& McCourt, 2006); Por outro lado, nas cerimónias necessárias à manutenção das relações sociais, quanto mais integrado o indivíduo está, mais as atitudes coletivas se sobrepõem às individuais no sentido de um consumo aumentado de bebidas alcoólicas (Reed, Lange, Ketchie \& Clapp, 2007). Dando um exemplo contrário, o acto de beber está frequentemente integrado em rituais afiliativos, em especial em grupos de grandes consumidores de álcool (Mohr, Averna, Kenny \& Del Boca, 2001).

Embora o papel das variáveis suporte social e autoregulação se mostrem de grande utilidade na análise empírica dos comportamentos de consumo de substâncias (nomeadamente o tabaco), são raros os estudos que relacionam conjuntamente estas variáveis (Ochsner, Luszczynska, Stadler, Knoll, Hornung \& Scholz, 2014).

\section{Metodologia}

Tomou-se o objectivo de investigar as relações entre suporte social, autorregulação e frequência de comportamentos de consumo de álcool, tabaco e outras substâncias psicoativas, em adultos portugueses. 
Trata-se de um estudo transversal, correlacional, comparativo e exploratório.

Os utentes que tiveram alta das consultas de psicologia, praticadas em diversas unidades de saúde, foram convidados a dar o seu consentimento para participar neste estudo. Estes não podiam apresentar transtornos mentais ou de comportamento, para além dos problemas foco de atenção clínica, segundo o DSM-V (American Psychiatric Association, 2014), que os motivaram a procurar a consulta [exemplo: Luto (V62.82), Problema de relação não especificado (V62.81), Problema de fase de vida / biográfico (V62.89)...]. Julgou-se que estes pacientes, aparentemente bem e ajustados perante a adversidade, são os que mais reconhecem o potencial promotor dos recursos de saúde e não hesitam em usá-los proactivamente em favor do seu bem-estar. Consequentemente, pensou-se que este contexto controlado de recolha de dados reunia as condições favoráveis à recolha de dados consentâneos com os objetivos deste estudo. A taxa de aceitação foi de $80 \%$, pelo que obteve-se, sequencialmente, uma amostra selecionada de 368 voluntários anónimos. Cerca de 5 sujeitos foram excluídos devido a não terem preenchido adequadamente os questionários. Por não assinalarem o consumo de qualquer substância, 23 inquéritos foram descartados.

Foram inquiridos 340 adultos saudáveis, dos quais $261(76.8 \%)$ eram mulheres e 79 (23.2\%) homens. O indivíduo mais novo tinha 18 anos e o mais velho, 76 anos $(M=39$; DP $=11)$. Dos respondentes $(n=$ 340), a esmagadora maioria pertencia à população ativa (81.8\% empregados), mais aqueles que se jubilaram (5.9\% reformados), outros $2.1 \%$ trabalhavam em casa, 9.7\% encontravam-se desempregados e somente $0.6 \%$ estavam de baixa. Quanto ao nível de formação académica dos respondentes, pouco mais de metade $(51.4 \%)$ frequentou graduações do nível superior; $30.9 \%$ situaram-se no ensino secundário; $16.2 \%$ colocaram-se nos ciclos de ensino básico e intermédios e apenas 1.5\% não completou o ensino básico.

Utilizaram-se os seguintes instrumentos:

Questionário Redurido de Suporte Social - SSQ6 (Sarason, Sarason, Shearin \& Pierce, 1987) constitui- se em seis itens, que avalia dois elementos básicos da perceção do suporte social: $\mathrm{O}$ número de pessoas que cada indivíduo percebe como estando disponíveis para o apoiarem e ajudarem numa determinada situação é avaliado de forma interrogativa e apresentado na primeira parte do item. Os indivíduos podem referir um número máximo de nove pessoas, sendo também possível responder "ninguém". O grau de satisfação com a globalidade do suporte percebido nessa mesma situação surge na segunda parte de cada item. Nesse sector, utiliza-se uma escala de tipo Likert, de seis pontos: desde muito insatisfeito (1) a muito satisfeito (6). Este dispositivo permite obter um índice de perceção de número de suporte disponível (SSQ6N) e um índice de perceção da satisfação com o suporte social disponível (SSQ6S). Os trabalhos efetuados de tradução, adaptação e sobre as características métricas da versão portuguesa do SSQ6 (Pinheiro, 2003; Pinheiro \& Ferreira, 2001, 2002) revelaram índices de consistência interna elevados $(\alpha \approx .90)$ para o instrumento, quer para a dimensão número (SSQ6N) quer para a dimensão satisfação (SSQ6S). Outros resultados destes estudos detalhados enfatizam a utilidade da conceção bidimensional da perceção de suporte social, na medida em que permite diferenciar os apoios esperados dos diferentes subsistemas sociais que rodeiam o indivíduo (por exemplo, família e amigos). Desta forma, poderá ser entendido como um indicador das relações positivas com os outros e da integração social versus isolamento social. Foram também encontradas correlações positivas entre as duas dimensões do SSQ6 com outras variáveis de bemestar psicológico, em especial com o desenvolvimento pessoal e a aceitação de si próprio. Neste estudo, seguiu-se a sugestão de Seco, Pereira, Dias, Casimiro e Custódio (2005) que consiste em pré-definir 9 entidades de suporte que registaram maior frequência de escolha no estudo de Pinheiro (2003), a fim de facilitar e garantir o preenchimento correto por parte dos respondentes.

Questionário Reduzido de Auto-regulação - SSRQ (Carey, Neal \& Collins, 2004) é constituído por 31 itens, concebido para a obtenção de um índice total de autorregulação, embora estudos posteriores (Neal 
\& Carey, 2005) sugiram uma estrutura de dois fatores: Controlo de impulsos e Estabelecimento de objetivos. Os sujeitos respondem aos itens assinalando o seu nível de concordância com as afirmações dos itens. Foi adaptado por García Del Castillo e Dias (2009) para conduzir os seus estudos junto da população portuguesa. Estes investigadores encontraram, na aplicação SSRQ a indivíduos com idades até aos 19 anos, uma boa estrutura factorial, com a maioria dos itens a apresentar saturações elevadas (genericamente superiores a .40) nos fatores a que pertencem. Também analisaram a consistência interna e encontraram um valor elevado de alfa total de .89 , variando entre os .82 na subescala Controlo de Impulsos e .85 na subescala Estabelecimento de Objetivos; A Escala de consumo de tabaco, alcohol y otras outras substâncias - ESCON (García del Castillo, López-Sánchez, Gázquez \& García del Castillo-López cit. Por García del Castillo, 2011) trata-se de um formulário de autoaplicação de vinte e quatro itens, de apresentação eclética e sobreponível, que parte do princípio de que a comparação entre estudos sobre os comportamentos de consumo de substâncias apresenta-se uma tarefa difícil, visto ainda não se ter atingido consenso, entre os pesquisadores, sobre as medidas a utilizar. Nesse sentido, este instrumento pretende constituir um dispositivo exploratório fácil de aplicar, que abarque todo o espectro de consumo e produtor de dados objetivos e suficientemente fiáveis para a avaliação do consumo de psicotrópicos.

Os objetivos desta investigação inscrevem-se na exploração das relações entre suporte social percebido e a autorregulação e respetivas variações consoante comportamentos face ao álcool, tabaco e outras substâncias psicoativas. Consequentemente, as hipóteses, que levantamos de seguida, assumem um carácter exploratório, tomando como ponto de partida o raciocínio indicado na fundamentação teórica deste estudo:

1. Existem correlações significativas entre $O$ Suporte Social e a Autorregulação.

2. Existem variação significativa no Suporte Social Percebido conforme o Consumo de tabaco, álcool e outras substâncias.
3. Existem variações significativas na Autorregulação conforme o Consumo de tabaco, álcool e outras substâncias. $\mathrm{Na}$ análise estatística, destacamos dois tipos de tratamento de dados: as correlações lineares para averiguar se existe associação entre duas variáveis numéricas e testes de igualdade de distribuição, para averiguar se existem diferenças entre grupos independentes.

\section{Resultados}

As medidas de consistência interna das escalas, na testagem desta amostra, revelaram-se adequadas, ultrapassando os valores dos estudos originais, em todos os fatores e na globalidade (alfa superior a .90).

Considerando que as escalas utilizadas resultam de somas de itens tipo Likert, que são variáveis numéricas e ordinais, utilizamos o coeficiente de correlação de Spearman e pelo mesmo motivo foi utilizamos o teste de Kruskal-Wallis na comparação de medidas de localização de mais de duas amostras independentes.

Tabela 1

Coeficientes de correlação de Spearman entre o suporte social $e$ a autorregulação

\begin{tabular}{lllc}
\hline & & \multicolumn{2}{c}{ Suporte Social (SSQ) } \\
\hline & SSQ6_N & SSQ6_S \\
& Auto-regulação & $.131^{*}$ & $.277^{* *}$ \\
$\begin{array}{l}\text { Autorregulação } \\
\text { (SSRQ) }\end{array}$ & $\begin{array}{l}\text { Estabelecimento } \\
\text { de objectivos }\end{array}$ & .106 & $.270^{* *}$ \\
& $\begin{array}{l}\text { Controlo de } \\
\text { impulsos }\end{array}$ & .105 & $.239^{* *}$ \\
\hline
\end{tabular}

${ }^{*} \mathrm{p}<.05 * * \mathrm{p}<.01$

$\mathrm{Na}$ tabela 1 observa-se como a percepção do número de pessoas disponíveis para oferecer suporte social (SSQ6N) correlaciona-se de maneira positiva e significativa com o fator de autorregulação. Esta correlação, ainda que significativa, deve ser considerada baixa. 
Por outro lado, a satisfação com o apoio social percebido apresenta-se correlacionado de forma positiva e significativa com os três fatores da escala de autorregulação. No caso das correlações com o fator "autorregulação" (.277) e com o fator "estabelecimento de objectivos" (.27) estas podem ser consideradas moderadas, já que em ambos os casos estão próximas de .30. A correlação com o fator "controlo de impulsos" mostra-se num nível moderado a baixo (.239), dado que se expressa num valor que é superior a .20 , mas inferior a .30 .

Entre os sujeitos da amostra que não fumam, a mediana da percepção do número de suporte social é de 2.0 contra 2.3 e 2.7 entre os que fumam ou fumam raramente, pelo que estas diferenças têm significado estatístico $(X=6.157$; $p=.046)$. Depreende-se que entre os sujeitos que não fumam a percepção do suporte social é inferior.
Detetaram-se diferenças significativas na escala de autorregulação total e na subescala controlo de impulsos relativamente ao consumo de tranquilizantes. A mediana da autorregulação entre sujeitos que não tomam tranquilizantes é de 114, entre os que o fazem raramente a mediana da autorregulação é inferior (112) e ainda menor (101) entre os que, sim, tomam tranquilizantes. Deduzimos que o consumo de tranquilizantes conotase com uma redução da autorregulação dos sujeitos. Especificamente, este resultado pode ser explicável pela variação achada no controlo de impulsos relativamente ao consumo de tranquilizantes. Entre os sujeitos que tomam tranquilizantes, o controlo de impulsos mediano é de 44, que aumenta para 53 entre os sujeitos que raramente tomam tranquilizantes e é de 54 entre os que não tomam tranquilizantes.

Tabela 2

Mediana das escalas de suporte social e autorregulação segundo a frequência de consumo de substâncias (Kruskal-Wallis)

\begin{tabular}{|c|c|c|c|c|c|c|}
\hline Mediana & \multicolumn{3}{|c|}{ Fuma? } & \multicolumn{3}{|c|}{ Toma tranquilizantes } \\
\hline & $\begin{array}{c}\operatorname{Sim} \\
(n=42)\end{array}$ & $\begin{array}{l}\text { Raramente } \\
(n=33)\end{array}$ & $\begin{array}{c}\text { Não } \\
(n=265)\end{array}$ & $\begin{array}{c}\operatorname{Sim} \\
(n=23)\end{array}$ & $\begin{array}{l}\text { Raramente } \\
(n=49)\end{array}$ & $\begin{array}{c}\text { Não } \\
(n=268)\end{array}$ \\
\hline \multirow{2}{*}{ SSQ6_N } & 2.3 & 2.7 & 2.0 & 1.7 & 2.2 & 2.1 \\
\hline & \multicolumn{3}{|c|}{$X=6.157 ; p=.046$} & \multicolumn{3}{|c|}{$X=2.246 ; p=.325$} \\
\hline \multirow{2}{*}{ SSQ6_S } & 5.3 & 5.2 & 5.2 & 5.0 & 5.3 & 5.2 \\
\hline & \multicolumn{3}{|c|}{$X=1.576 ; p=.455$} & \multicolumn{3}{|c|}{$X=1.406 ; p=.495$} \\
\hline \multirow{2}{*}{$\begin{array}{l}\text { Autorregulação } \\
\text { total (SSRQ) }\end{array}$} & 112.5 & 110.0 & 114.0 & 101.0 & 112.0 & 114.0 \\
\hline & \multicolumn{3}{|c|}{$\mathrm{X}=4.799 ; p=.091$} & \multicolumn{3}{|c|}{$X=6.316 ; p=.043$} \\
\hline \multirow{2}{*}{$\begin{array}{l}\text { Estabelecimento de } \\
\text { objectivos (SSRQ) }\end{array}$} & 61.5 & 59.0 & 60.0 & 58.0 & 58.5 & 60.0 \\
\hline & \multicolumn{3}{|c|}{$\mathrm{X}=1.998 ; p=.368$} & \multicolumn{3}{|c|}{$\mathrm{X}=3.489 ; p=.175$} \\
\hline \multirow{2}{*}{$\begin{array}{l}\text { Controlo de } \\
\text { impulsos (SSRQ) }\end{array}$} & 53.0 & 52.0 & 54.0 & 44.0 & 53.0 & 54.0 \\
\hline & \multicolumn{3}{|c|}{$X=5.525 ; p=.063$} & \multicolumn{3}{|c|}{$\mathrm{X}=7.787 ; p=.02$} \\
\hline
\end{tabular}




\section{Discussão}

A primeira hipótese considera uma relação entre o suporte social percebido e a autorregulação. Efetivamente, esta é mantida por ligações positivas, débeis, mas significativas entre o suporte social e a de autorregulação. Não obstante, a autorregulação parece estar mais vinculada à satisfação com o suporte social, comparativamente com o número de figuras provedoras de suporte social.

A observação destas ligações estatísiticas pode contribuir para a ideia que, mais do que a quantidade de pessoas na rede social, é a satisfação com a qualidade do apoio instrumental e emocional que se mostra mais em linha com a capacidade dos sujeitos se autorregularem. Estes achados podem corroborar as teorias de vinculação e de corregulação social.

Atualmente tem havido grande interesse em saber se as experiências relacionais têm influência no desenvolvimento neurocognitivo (em particular, das funções) desde a infância à adultícia, tanto a nível funcional quanto estrutural (Nelson \& Bloom, 1997). Os investigadores das teorias da vinculação têm sugerido que os cuidadores primários, enquanto suporte social primordial, atuam inicialmente como reguladores externos do afeto e tempo da criança e que depois vão fomentando a competência de autorregulação desta até à maioridade (Hofer, 1995; Spangler, Schieche, Ilg, Maier \& Ackermann, 1994).

Sarason et al. (1987) evocam o preceituado por Bowlby, referindo que da mesma maneira que as necessidades de vinculação na criança não se resolvem no simples recebimento de cuidados, a demanda de afeição, segurança, aceitação não se esgota nos primeiros anos de vida. Os adultos dependem das relações sociais para continuarem se abastecer de amor, confiança e valorização e nesse sentido, o apoio social funciona, na maioridade, como uma extensão ou contrapartida das experiências de vinculação da infância. Consequentemente, olhando à literatura sobre esta matéria, a rede de suporte social pode atuar com a continuação ou a compensação, na idade adulta, dos fenómenos de vinculação fundamentais para a saudável maturação cerebral e desenvolvimento mental ocorridos (ou não) na primeira infância. Assim, interpretando, à luz das teorias enunciadas, os dados deste estudo, é lícito pensar os sujeitos que beneficiam de uma rede social de suporte robusta, gratificante e contentora, que satisfaz as suas necessidades de segurança, afeição e valorização possam mostra-se mais competentes para se autorregular.

Tomando uma análise interpretativa alternativa, unicamente baseada nos achados desta investigação, também se pode especular se o grupo social pressiona a motivação do sujeito para adquirir estratégias de autorregulação sob pena de, se não o fizer, ser ostracizado. Assim, é razoável pensar que os sujeitos que mais sabem autorregular-se mostram-se mais cooperantes, asseguram a sua afiliação no grupo e beneficiam e gratificam-se com o seu apoio. Por outro lado, os sujeitos menos aptos para se autorregularem, sobrecarregam o grupo até serem progressivamente rejeitados pelo mesmo, o que repercute-se em menor número de figuras significativas mas, sobretudo, em satisfação mais reduzida com o suporte social.

Respondendo à segunda hipótese, esta propõe uma variação significativa do suporte social percebido em função do consumo de tabaco, álcool e outras substâncias. Esta é legitimada por diferenças significativas na mediana do número de figuras providentes de suporte social relativamente ao consumo de tabaco. Constata-se tendência semelhante, embora não significativa, relativamente ao álcool. Os dados deste estudo rementem para a ideia de que os fumadores ocasionais parecem beneficiar de um suporte social mais numeroso do que os não fumadores e os fumadores. Estes resultados contrariam o efeito protetor do suporte social relativamente ao consumo moderado de nicotina (Carter-Sowell, Chen \& Williams, 2008). É possível que isso se suceda, segundo a literatura, porque os fumadores (em particular os ocasionais) andam mais envolvidos em rituais afiliativos, o que resulta numa rede social mais numerosa. Nestas cerimónias, é expectável que exista uma sinergia consolidada entre o uso de tabaco e o uso de álcool (Falk, Yi \& HillerSturmhofel, 2006). Tal não implica maior satisfação 
relativamente ao apoio providenciado pela rede social, pois sobre esta variável, não se observam neste estudo quaisquer influências dignas de registo provenientes do consumo de álcool, tabaco e outras substâncias. Isso é indicativo de que o uso de substâncias psicoativas pode estar ligado a redes sociais mais abrangentes, mas provavelmente mais superficiais e menos satisfatórias. Além disso, o medo de ostracismo pode incrementar os consumos que, por sua vez, retroalimentam as relações sociais mas não necessariamente geradoras de suporte social de qualidade (Wagner, Burg \& Sirois, 2004).

Atendendo à terceira e última hipótese, esta implica uma modificação significativa da autorregulação conforme o consumo de tabaco, álcool e outras outras substâncias. A auto-regulação surge menor entre os consumidores de tranquilizantes, o que remete para os efeitos adversos decorrentes da toma destes medicamentos. Esta disposição é ratificada pelas diferenças significativas nas medianas das subescalas de autorregulação e do controlo de impulsos, que são tanto maiores quanto menor se apresenta a frequência de uso de tranquilizantes. Por outro lado, "nervosismo" é um termo ambíguo frequentemente usado em queixas dos doentes ao seu médico assistente. Com efeito, esta expressão desdobra-se em significações eufemísticas que vão para além do sinónimo "ansiedade", como: "agitação"; "impulsividade"; "irritação"; "instabilidade" ou mesmo "leviandade". Logo, não é de estranhar que possam ocorrer fenómenos de automedicação ou erros diagnósticos e prescrições indevidas de ansiolíticos a sujeitos mais extrovertidos, desinibidos e impulsivos portanto, com menores níveis de auto-regulação.

A escassez de diferenças significativas nos níveis de autorregulação quanto à frequência de uso de substâncias poder-se-á dever às taxas de consumo muito baixas entre os respondentes, o que segundo García Del Castillo e Dias (2009), pode intervir na definição última dos resultados.

A amostra deste estudo apresenta-se demasiado pequena, selecionada e heterogénea para figurar-se representativa da população. Tais restrições impossibilitam a generalização dos resultados. Acresce que as taxas de consumo entre os respondentes figuram-se muito baixas, o que segundo García Del Castillo e Dias (2009), pode condicionar questionavelmente a apresentação dos achados. As relações estatisticamente significativas encontradas entre as variáveis mostram-se demasiado fracas para permitir o uso de equações estruturais, o que constrange a averiguação de causalidades.

As relações encontradas entre a autorregulação e o suporte social, bem como a constatação das suas oscilações em torno de condutas de consumo de substâncias psicoactivas em adultos, remete para a necessidade de continuar a explorar a pertinência explicativa destas variáveis em futuros estudos sobre os comportamentos de uso de substâncias psicoativas. Considera-se que potencial preventivo desta linha investigativa, assim o justifica

\section{Agradecimentos}

À Agência Regional para o Desenvolvimento da Investigação Tecnologia e Inovação, pelo financiamento deste projeto n. ${ }^{\circ} 1974$;

Ao Serviço de Saúde da Região Autónoma da Madeira, E.P.E, pela cooperação que estabeleceu com esta iniciativa investigativa.

\section{References}

American Psychiatric Association. (2014). Manual de diagnóstico e estatística das Perturbações Mentais: DSM-V (5 edição). Lisboa: Climepsi.

Baumeister, R. F., Vohs, K. D., \& Tice, D. M. (2007). The strength model of self-control. Current Directions in Psychological Science, 16(6), 351-355.

Busch, H., \& Hofer, J. (2012). Self-regulation and milestones of adult development: Intimacy and generativity. Developmental Psychology, 48(1), 282-293.

Carey, K. B., Neal, D. J., \& Collins, S. E. (2004). A psychometric analysis of the Self-Regulation Questionnaire. Addictive Behaviors, 29(2), 253-260.

Carter-Sowell, A. R., Chen, Z., \& Williams, K. D. (2008). Ostracism increases social susceptibility. Social Influence, 3(3), 143-153. 
Carver, C. S., \& Scheier, M. F. (1998). On the self-regulation of behavior. New York: Cambridge University Press.

Carver, C. S. \& Scheier, M. F. (1982). Control theory: A useful conceptual framework for personalitysocial, clinical, and health psychology. Psychological Bulletin, 92(1), 111-135.

Cullum, J., O’Grady, M., Sandoval, P., Armeli, S., \& Tennen, H. (2013). Ignoring norms with a little help from my friends: Social support reduces normative influence on drinking behavior. Journal of Social and Clinical Psychology, 32(1), 17-33.

Falk, D. E., Yi, H. Y., \& Hiller-Sturmhofel, S. (2006). An epidemiologic analysis of co-occurring alcohol and tobacco use and disorders: findings from the National Epidemiologic Survey on alcohol and Related Conditions. Alcohol Research \& Health, 29(3), 162-71.

Fujita, K., \& MacGregor, K. E. (2012). Basic goal distinctions. In H. Aarts, \& A. J. Elliot (Eds.), Goaloriented behavior (pp. 85-114). New York: Psychology Press/Taylor \& Francis.

García del Castillo, J. A., \& Dias, P. C. (2009). Autoregulação, resiliência e consumo de substâncias na adolescência: contributos da adaptação do questionário reduzido de auto-regulação. Psicologia, Saúde e Doenças, 10(2), 205-216.

García del Castillo, J. A. (2011). La evaluación del consumo de drogas en perspectiva. Health and Addictions, 11(1), 7-15.

Glantz, M. D., \& Leshner, A. I. (2000). Drug abuse and developmental psychopathology. Development and Psychopathology, 12(4), 795-814.

Goetz, J. L., Keltner, D., \& Simon-Thomas, E. (2010). Compassion: An evolutionary analysis and empirical review. Psychological Bulletin, 136(3), 351-374.

Hofer, M. A. (1995). Hidden regulators: Implication for a new understanding of attachment, separation, and loss. In S. Golberg, R. Muir, \& J. Kerr (Eds.), Attachment theory: Social, developmental, and clinical perspectives (pp. 203230). Hillsdale, NJ: The Analytic Press.
Mischel, W., Cantor, N., \& Feldman, S. (1996). Principles of self-regulation: the nature of willpower and selfcontrol. In E.T. Higgins, \& A.W. Kruglanski (Eds.), Social Psychology: Handbook of basic principles (pp.329360). New York: Guilford Press.

Mohr, C. D., Averna, S., Kenny, D. A., \& Del Boca, F. K. (2001). Getting by (or Getting High) with a little help from my friends: An examination of adult alcoholics' friendships. Journal of Studies on Alcohol, 62(5), 637-646.

Neal, D. J., \& Carey, K. B. (2005). A Follow-Up Psychometric Analysis of the Self-Regulation Questionnaire. Psychology of Addictive Behaviors, 19(4), 414-422.

Nelson, C. A., \& Bloom, F. E. (1997). Child development and neuroscience. Child Development, 68(5), 970-987. doi: 10.1111/j.1467-8624.1997.tb01974.x

Norcross, J. C., Ratzin, A. C., \& Payne, D. (1989). Ringing in the New Year: The change processes and reported outcomes of resolutions. Addictive Behaviors, 14(2), 205-212.

Ochsner, S., Luszczynska, A., Stadler, G., Knoll, N., Hornung, R., \& Scholz, U., (2014). The interplay of received social support and self-regulatory factors in smoking cessation. Psychology \& Health, 29(1), 16-31.

Pinheiro, M. R. M. (2003). Uma época especial: suporte social e vivências académicas na transição e adaptação ao ensino superior. (Tese de Doutoramento não publicada). Faculdade de Psicologia e de Ciências da Educação da Universidade de Coimbra, Coimbra.

Pinheiro, M. R., \& Ferreira, J. A. (2001). Avaliação do suporte social em contexto do Ensino superior. In Actas do V Seminário de Investigação e Intervenção Psicológica no Ensino Superior. Viana do Castelo: Instituto Politécnico de Viana do Castelo.

Pinheiro, M. R., \& Ferreira, J. A. (2002). O questionário de suporte social: Adaptação e validação da versão portuguesa do Social Support Questionnaire (SSQ6). Psychologica, 30, 315-333. 
Reed, M. B., Lange, J. E., Ketchie, J. M., \& Clapp, J. D. (2007). The relationship between social identity, normative information and college student drinking. Social Influence, 2(4), 269-294.

Reifman, A., Watson, W.K., \& McCourt, A. (2006). Social Networks and College Drinking: Probing Processes of Social Influence and Selection. Personality and Social Psychology Bulletin, 32(6), 820-832.

Sarason, B. R., Sarason, I. G., Shearin, E. N., \& Pierce, G.R. (1987). A brief measure of social support: Practical and theoretical implications. Journal of Social and Personal Relationships, 4(4), 497-510.

Sarason, B. R., Sarason, I. G., \& Pierce, G. R. (1990). Traditional views of social support and their impact on assessment. In B. R. Sarason, I. G. Sarason \& G.R. Pierce (Eds.), Social Support: An Interactional View (pp. 9-25). New York: Wiley.

Seco, G., Pereira, I., Dias, I., Casimiro, M., \& Custódio, S. (2005). Para uma abordagem psicológica da transição do ensino secundário para o ensino superior: Pontes e alçapões. Psicologia e Educação, IV , 7-21.
Spangler, G., Schieche, M., Ilg, U., Maier, U., \& Ackermann, C. (1994). Maternal sensitivity as an external organizer for biobehavioral regulation in infancy. Developmental Psychobiology, 27(7), 425-437.

Thoits, P. (1986). Conceptual, methodological, and theoretical problems in studying social support as a buffer against life stress. Journal of Health and Social Behavior, 23(2), 145-159.

Wagner, J., Burg, M., \& Sirois, B. (2004). Social support and the transtheoretical model: Relationship of social support to smoking cessation stage, decisional balance, process use, and temptation. Addictive Behaviors, 29(5), 1039-1043.

Zimmerman, B. J. (2000). Attainment of self-regulation: A social cognitive perspective. In M. Boekaerts, P. R. Pintrich, \& M. Zeidner (Eds.), Handbook of self-regulation (pp. 13-39). San Diego, CA: Academic Press.

Recibido: 31 de maio de 2016 Aceptado: 26 de outubro de 2016 
